\title{
Contributions to the Gaussian Line Broadening of the Proxyl Spin Probe EPR Spectrum Due to Magnetic-Field Modulation and Unresolved Proton Hyperfine Structure
}

\author{
Barney L. Bales,* Miroslav Peric, ${ }^{*}$ and Maria Teresa Lamy-Freund $\dagger$ \\ *Department of Physics and Astronomy and The Center for Cancer and Developmental Biology, California State University at Northridge, \\ Northridge, California 91330; and †Instituto de Física, Universidade de São Paulo, Caixa Postal 66318, 05315-970 São Paulo, Brazil
}

Received July 10, 1997; revised January 9, 1998

\begin{abstract}
A simple expression is derived to compute the total $\mathbf{G}$ aussian linewidth of a $\mathrm{V}$ oigt line that is broadened by sinusoidal magneticfield modulation as follows: $\Delta H_{\mathrm{pp}}^{\mathrm{G}}\left(H_{\mathrm{m}}\right)^{2}=\Delta H_{\mathrm{pp}}^{\mathrm{G}}(0)^{2}+\kappa^{2} H_{\mathrm{m}}^{2}$,
\end{abstract} where $\Delta H_{\mathrm{pp}}^{\mathrm{G}}\left(H_{\mathrm{m}}\right)$ is the Gaussian linewidth observed with an modulation amplitude $H_{\mathrm{m}} / 2$ and $\Delta H_{\mathrm{pp}}^{\mathrm{G}}(0)$ is the Gaussian linewidth in the limit of zero modulation. The field modulation contributes an additional G aussian broadening of $\kappa H_{m}$, where $\kappa$ is a constant, which adds in quadrature to $\Delta H_{\mathrm{pp}}^{\mathrm{G}}(0)$ to give the total $\mathrm{G}$ aussian linewidth. Denoting the overall linewidth of the Voigt line in the absence of modulation broadening by $\Delta H_{\mathrm{pp}}^{0}(0)$, it is shown, both by analytical means and by spectral simulation, that the constant $\kappa$ is equal to $1 / 2$ in the limit of $H_{\mathrm{m}} \ll \Delta H_{\mathrm{pp}}^{0}(0)$; however, using values of $H_{\mathrm{m}}$ as large as $\Delta H_{\mathrm{pp}}^{0}(0)$ leads to only minor departures from $\kappa=1 / 2$. The formulation is valid both for Lorentzian and Voigt lines and is tested for 2,2,5,5-tetramethylpyrrolidin-1-oxyl-3-carboxylic acid (3-carboxy proxyl) in $\mathrm{CCl}_{4}$ and in aqueous buffer. This spin probe was studied because the proxyl group is the only major spin-probe moiety whose $G$ aussian linewidth had not been characterized in the literature. For 3-carboxy proxyl, it is found that $\Delta H_{\mathrm{pp}}^{\mathrm{G}}(0)=1.04 \pm 0.01 \mathrm{G}$ independent of solvent polarity. Precision values of the ${ }^{14} \mathrm{~N}$ hyperfine coupling constant for 3-carboxy proxyl at $9.5^{\circ} \mathrm{C}$ are as follows: $14.128 \pm$ $0.001 \mathrm{G}$ in $\mathrm{CCl}_{4}$ and $16.230 \pm 0.002 \mathrm{G}$ in aqueous buffer. The temperature dependence of $\Delta H_{\mathrm{pp}}^{\mathrm{G}}(0)$ and the ${ }^{14} \mathrm{~N}$ hyperfine coupling constant are reported as empirical equations. Results of the present work taken together with previously published data permits accurate correction for the effects of inhomogeneous broadening due to unresolved hyperfine structure and modulation broadening for the majority of spin probes in common use. $\odot 1998$ Academic Press

\section{INTRODUCTION}

To increase the signal-to-noise ratios of EPR spectra, most conventional continuous-wave spectrometers employ magneticfield modulation (1). Modulation distorts the signal, leading to modulation broadening, which has traditionally led the experimentalist to seek a modulation amplitude which is a compromise between noise and resolution. Alternative approaches avoiding field modulation have also been proposed (2-5). In most nitroxide spin-probe studies, resolution is not an issue ${ }^{1}$; however, in many studies, determination of the correct intrinsic (Lorentzian) linewidth is important. For these types of studies, until recently (7), the only recourse had been to employ a small modulation amplitude in order to obtain a faithful reproduction of the lineshape. For most spin probes, the relevant lineshape is a Lorentzian-Gaussian convolution (Voigt), the Gaussian component occurring chiefly because of unresolved hyperfine structure (8). Recent work (7) showed that a modulationbroadened Voigt shape continues to be well described by the Voigt shape such that only the Gaussian component is broadened, leaving the Lorentzian component unchanged. The significance of this finding is that approximate deconvolution procedures developed to correct Voigt lines for unresolved hyperfine structure $(8)$ could also be used to correct for modulation broadening. Here, we find that rather large modulation amplitudes, of the order of the Voigt linewidth, may be employed without compromising the need to obtain accurate intrinsic widths. While predicting that only the Gaussian component was broadened, the previous work (7) fell short of providing a theory which explicitly yielded the Gaussian broadening as a function of the modulation amplitude. To provide such a theory is one purpose of this work.

A second purpose of the work is to characterize the Gaussian component of the proxyl group since it is the only major spin-probe moiety that has not been so characterized $(8,9)$. To achieve this end and to test the modulation-broadening theory experimentally, we studied the nitroxide spin probe 2,2,5,5tetramethylpyrrolidin-1-oxyl-3-carboxylic acid (3-carboxy proxyl) as a function of modulation amplitude and temperature in a buffered aqueous solution and in $\mathrm{CCl}_{4}$.

\section{EXPERIMENTAL}

The spin probe 3-carboxy proxyl (Synvar, Palo Alto, California) and carbon tetrachloride (Merck) were used as re-

\footnotetext{
${ }^{1}$ A notable exception occurs when the spin probe partitions between environments of different polarity leading to two overlapping spin-probe spectra. See for example, Fig. 1 of Ref. (6).
} 
ceived. Double distilled, deionized water was used to prepare Hepes (Sigma) buffer, pH 7.4. Solutions were prepared by weight and diluted to a final spin-probe concentration of $10^{-4}$ M. Some samples were deoxygenated by bubbling nitrogen gas through the solution for $30 \mathrm{~min}$. Air-saturated samples or deoxygenated samples were sealed into $50-\mu \mathrm{L}$ disposable pipettes which were housed in a quartz tube inside the variable temperature dewar. The temperature was controlled to about \pm $0.2 \mathrm{~K}$ by the Bruker Variable Temperature Unit BVT 2000 and was measured with a Fluke $51 \mathrm{~K} / \mathrm{J}$ thermometer with the probe placed just above the cavity. The temperature difference between the probe position and the sample was calibrated in a separate run. The magnetic field was measured with a Bruker ER 035 M NMR Gaussmeter. The field sweep was reproducible to within $0.0015 \mathrm{G}$ during any one experiment.

\section{THEORY}

\section{Unresolved Hyperfine Structure}

Unresolved hyperfine structure is well known to broaden EPR lines inhomogeneously (8). If a sufficiently large number of magnetic nuclei contribute to the broadening, they add Gaussian character to the EPR lineshape (8). For protons, the peak-to-peak Gaussian linewidth in a first-derivative spectrum $\Delta H_{\mathrm{pp}}^{\mathrm{G}}$ is given by $(8)$

$$
\left[\Delta H_{\mathrm{pp}}^{\mathrm{G}}\right]^{2}=\alpha \sum n_{j} a_{j}^{2},
$$

where $a_{j}$ is the unresolved proton hyperfine coupling constant (magnetic field units) due to $n_{j}$ equivalent protons in set $j$. The constant $\alpha$ was previously introduced (8) to take into account the difference in the actual Gaussian linewidth and that calculated from the second moment. For large numbers of interacting protons, the observed EPR spectral line is well approximated by a Voigt line shape, a Lorentzian-Gaussian convolution; the only known exception arises if the hyperfine coupling to single proton is much larger than the hyperfine couplings to all of the other protons (10). The Voigt shape is determined by a single parameter, $\chi$, as follows:

$$
\chi=\Delta H_{\mathrm{pp}}^{\mathrm{G}} / \Delta H_{\mathrm{pp}}^{\mathrm{L}},
$$

where $\Delta H_{\mathrm{pp}}^{\mathrm{L}}$ is the Lorentzian linewidth which is usually the quantity of interest, arising as it does from spin relaxation processes. The convolution produces a spectral line of observed linewidth $\Delta H_{\mathrm{pp}}^{0}$. It has been shown (8) that parameters measured from EPR spectra may be corrected accurately in a wide range of spin probe applications once $\chi$ has been determined. The power of this approach is that, within the limits of its applicability, all nitroxides may be treated universally. As is expected, a universal treatment begins to break down at large values of $\chi$, the region of large proton couplings (8).

Experimentally, there have been two approaches to find the parameter $\chi$. The first consists of measuring the relative heights of the wings of the spectral line, the so-called four-point method (11). See Figs. 11 and 12 of Ref. (8). The second involves least-squares fitting (12) of the spectral line to a sum of Gaussian and Lorentzian shapes taking advantage of the fact that such a sum is an excellent approximation to a Voigt $(8,13)$. Thus, the sum function

$$
Y^{\prime}(H)=I\left[\eta_{\mathrm{A}} L^{\prime}(H)+\left(1-\eta_{\mathrm{A}}\right) G^{\prime}(H)\right]
$$

is either equated to the experimental spectral line at the four points (8) or it is least-squares fit over a fit window (12). In Eq. [3], $Y^{\prime}(H)$ is the first derivative spectral line of linewidth $\Delta H_{\mathrm{pp}}^{0}$, $L^{\prime}(H)$ is a Lorentzian shape, $G^{\prime}(H)$ is a Gaussian shape, and $\eta_{\mathrm{A}}$ is the mixing parameter. The subscript $A$ indicates that $\eta_{\mathrm{A}}$ is the fraction of Lorentzian character based upon the doubly integrated spectral line (area). Both $L^{\prime}(H)$ and $G^{\prime}(H)$ are normalized to unit area and both have the same linewidth, equal to that of $Y^{\prime}(H), \Delta H_{\mathrm{pp}}^{0}$. Detailed expressions for $L^{\prime}(H)$ and $G^{\prime}(H)$ are given in Eq. [13] of Ref. (8). Once $\eta_{\mathrm{A}}$ is determined from least-squares fitting or the four-point method, a map from $\eta_{\mathrm{A}}$ to $\chi$ is needed to proceed. Such maps based upon the Voigt shape, which we refer to as the Voigt maps, are given by Eq. [9] of Ref. (8) or Eq. [8] of Ref. (12).

As expected, at large values of $\chi$, the detailed spectra for different spin probes begin to deviate significantly from one another (8). Except for the case of a single large proton coupling (10), these spectra are still well describe by the Voigt shape; however, the Voigt map is no longer accurate. A model hyperfine pattern called the "Universal Nitroxide" was introduced (8) which was a compromise pattern intermediate between many common spin probes. This produced a different map from $\eta_{\mathrm{A}}$ to $\chi$, given by Eqs. [14] and [10] of Ref. (8), which we refer to as the universal map. Later work showed (9) that the universal map was accurate all the way up to $\chi=5$ for doxyl-labeled $n$-alkyl chains.

Both the Voigt and universal maps were designed to give reasonable results over a wide range of $\chi$, compromising precision at small values of $\chi$ where corrections to find $\Delta H_{\mathrm{pp}}^{\mathrm{L}}$ are less critical because $\Delta H_{\mathrm{pp}}^{\mathrm{G}}$ is small anyway. If one is interested in $\Delta H_{\mathrm{pp}}^{\mathrm{G}}$ itself, as we are here, then a more accurate map is needed at low values of $\chi$. Such a map is given in Eq. [12] of the Appendix.

\section{Modulation Broadening}

We discuss the common case in which the magnetic field is modulated at an angular frequency $\omega_{\mathrm{m}}$ and is detected at the first harmonic producing first-derivative lineshapes (1). The modulation field is given by

$$
H(t)=\frac{H_{\mathrm{m}}}{2} \sin \left(\omega_{\mathrm{m}} t\right),
$$


where $H_{\mathrm{m}}$, often referred to as the modulation "'amplitude," ' is actually the peak-to-peak value.

We combine the following two facts: First, only the Gaussian component is broadened when a Voigt line is subjected to the modulation given by Eq. [4] (7); and second, the Gaussian linewidth is proportional to the root-mean-square moment of the inhomogeneous broadening (8). The second moment of Eq. [4] is given by $H_{\mathrm{m}}^{2} / 8$; thus, we write the modulation-induced Gaussian linewidth as $\kappa H_{\mathrm{m}}$, the constant $\kappa$ to be determined. The behavior of a Voigt line may be deduced by recalling that Gaussian linewidths add as the sum of their squares (8):

$$
\Delta H_{\mathrm{pp}}^{\mathrm{G}}\left(H_{\mathrm{m}}\right)^{2}=\Delta H_{\mathrm{pp}}^{\mathrm{G}}(0)^{2}+\kappa^{2} H_{\mathrm{m}}^{2},
$$

where $\Delta H_{\mathrm{pp}}^{\mathrm{G}}\left(H_{\mathrm{m}}\right)$ is the Gaussian linewidth observed with modulation amplitude $H_{\mathrm{m}} / 2$ and $\Delta H_{\mathrm{pp}}^{\mathrm{G}}(0)$ is the Gaussian linewidth in the limit of zero modulation. For a line that is Lorentzian in the absence of modulation broadening, $\Delta H_{\mathrm{pp}}^{\mathrm{G}}(0)$ $=0$ and Eq. [5] predicts that $\Delta H_{\mathrm{pp}}^{\mathrm{G}}\left(H_{\mathrm{m}}\right)$ would increase linearly with $H_{\mathrm{m}}$.

Analytical evaluation of $\kappa$. The value of $\kappa$ may be deduced from the closed analytical expression due to Wahlquist (14) for the linewidth of a modulation-broadened Lorentzian as follows:

$$
\left\{\frac{\Delta H_{\mathrm{pp}}^{0}\left(H_{\mathrm{m}}\right)}{\Delta H_{\mathrm{pp}}^{\mathrm{L}}}\right\}^{2}=\left\{\frac{H_{\mathrm{m}}}{\Delta H_{\mathrm{pp}}^{\mathrm{L}}}\right\}^{2}+5-2 \sqrt{4+\left\{\frac{H_{\mathrm{m}}}{\Delta H_{\mathrm{pp}}^{\mathrm{L}}}\right\}^{2}},
$$

where $\Delta H_{\mathrm{pp}}^{0}\left(H_{\mathrm{m}}\right)$ is the overall broadened linewidth and $\Delta H_{\mathrm{pp}}^{\mathrm{L}}$ is the (constant) Lorentzian linewidth. The overall linewidth may be decomposed into its Lorentzian and Gaussian components using the simple relationship for the Voigt shape discovered by Dobryakov and Lebedev (15),

$$
\left\{\frac{\kappa H_{\mathrm{m}}}{\Delta H_{\mathrm{pp}}^{0}\left(H_{\mathrm{m}}\right)}\right\}^{2}+\frac{\Delta H_{\mathrm{pp}}^{\mathrm{L}}}{\Delta H_{\mathrm{pp}}^{0}\left(H_{\mathrm{m}}\right)}=1,
$$

where the modulation-induced Gaussian linewidth is explicitly written $\kappa H_{\mathrm{m}}$. Substituting $\Delta H_{\mathrm{pp}}^{0}\left(H_{\mathrm{m}}\right)$ from Eq. [6] into Eq. [7] and expanding for small values of $H_{\mathrm{m}} / \Delta H_{\mathrm{pp}}^{\mathrm{L}}$ yields $\kappa=1 / 2$. Strictly speaking, this expansion ought to be valid only for a Lorentzian line; however, if we think of a Voigt line as comprising a set of Lorentzian lines imposed upon a hyperfine pattern determined by the unresolved hyperfine structure, then the additional Gaussian broadening will still be given by Eq. [5]. Conceptually, the expansion leading to $\kappa=1 / 2$ ought to extend only to small values of $H_{\mathrm{m}} / \Delta H_{\mathrm{pp}}^{\mathrm{L}}$; however, we next find by numerical computation that this limitation is unnecessarily restrictive.

Numerical evaluation of $\kappa$. Previously (7), plots of $\Delta H_{\mathrm{pp}}^{\mathrm{G}}\left(H_{\mathrm{m}}\right)$ vs $H_{\mathrm{m}}$ for Lorentzian lines were found to be approximately linear as is predicted by Eq. [5]; however, the curves did not extrapolate to the origin. This difficulty may be traced to the inadequacy of the previous map with respect to the Gaussian component at small values of $\chi$. An accurate map for $\chi<0.5$ is given in Eq. [12] of the Appendix.

Lorentzian and Voigt line shapes with input values of $\chi=$ 0.1 to 4.0 , under the influence of field modulation, were simulated as described in Ref. (7). These were fit (12) to Eq. [3] using a fit window of $4.0 \Delta H_{\mathrm{pp}}^{0}(0)$. For values of $\chi<0.5$, the map in Eq. [12] of the Appendix and for values of $\chi>0.5$ the universal map (Eqs. [14] and [10] of Ref. (8)) were used to find the best fit values of $\chi$. The Dobryakov-Lebedev relation (15) was then used to derive values of $\Delta H_{\mathrm{pp}}^{\mathrm{G}}\left(H_{\mathrm{m}}\right)$ and $\Delta H_{\mathrm{pp}}^{\mathrm{L}}$.

To facilitate comparison of the modulation-induced Gaussian broadening at different values of $\chi$, we rearrange Eq. [5] and divide by the unbroadened overall linewidth, $\Delta H_{\mathrm{pp}}^{0}(0)$, as follows:

$$
\frac{\Delta H_{\mathrm{pp}}^{\mathrm{G}^{*}}}{\Delta H_{\mathrm{pp}}^{0}(0)}=\frac{\sqrt{\Delta H_{\mathrm{pp}}^{\mathrm{G}}\left(H_{\mathrm{m}}\right)^{2}-\Delta H_{\mathrm{pp}}^{\mathrm{G}}(0)^{2}}}{\Delta H_{\mathrm{pp}}^{0}(0)}=\kappa \frac{H_{\mathrm{m}}}{\Delta H_{\mathrm{pp}}^{0}(0)} .
$$

Here, the first equality defines $\Delta H_{\mathrm{pp}}^{\mathrm{G}^{*}}$. The left-hand side of Eq. [8] is the normalized Gaussian broadening, which, according to the right-hand side ought to be a linear function of the normalized peak-to-peak modulation amplitude. For a Lorentzian line, $\Delta H_{\mathrm{pp}}^{\mathrm{G}}(0)=0$ and Eq. [8] reduces to a linear relation between the Gaussian linewidth and $H_{\mathrm{m}}$.

Figure 1 shows plots of the left-hand side of Eq. [8] (Fig. 1a) and the normalized values of $\Delta H_{\mathrm{pp}}^{\mathrm{L}}$ (Fig. 1b) as functions of the normalized modulation amplitude. The dashed lines in each case represent the values derived from fitting the simulated spectra; the upper corresponding to $\chi=0.1$ and the lower to $\chi$ $=4.0$. The solid lines are plots of the theory: Eq. [8] with $\kappa=$ $1 / 2$ (Fig. 1a), and constant $\Delta H_{\mathrm{pp}}^{\mathrm{L}}$ (Fig. 1b). Interestingly, the deviation from the prediction of Eq. [8] with $\kappa=1 / 2$ is less at higher values of $\chi$. Note that in Fig. $1, H_{\mathrm{m}}$ is allowed to attain a maximum value equal to the unbroadened linewidth; that is, in practice, the limitation that $H_{\mathrm{m}} \ll \Delta H_{\mathrm{pp}}^{0}(0)$ may be relaxed to $H_{\mathrm{m}} \approx \Delta H_{\mathrm{pp}}^{0}(0)$. Given the uncertainties in most experiments, discussed on p. 77 of Ref. (8), the error in the Gaussian broadening calculated from Eq. [5] is likely to be less than other uncertainties. It should be borne in mind that one of these uncertainties is the choice of map at values of $\chi>2$ (8). In case more precision is merited, a more accurate value of $\kappa$ may be found by fitting each curve in Fig. 1a to a quadratic function of $H_{\mathrm{m}} / \Delta H_{\mathrm{pp}}^{0}(0)$ and expressing the second-order coefficient as a function of $\chi$. This yields

$$
\kappa=1 / 2+0.068 \exp (-0.658 \chi) \frac{H_{\mathrm{m}}}{\Delta H_{\mathrm{pp}}^{0}(0)} .
$$

Equation [9] reflects the fact observed earlier that the deviation of $\kappa$ from $1 / 2$ diminishes at higher values of $\chi$. 

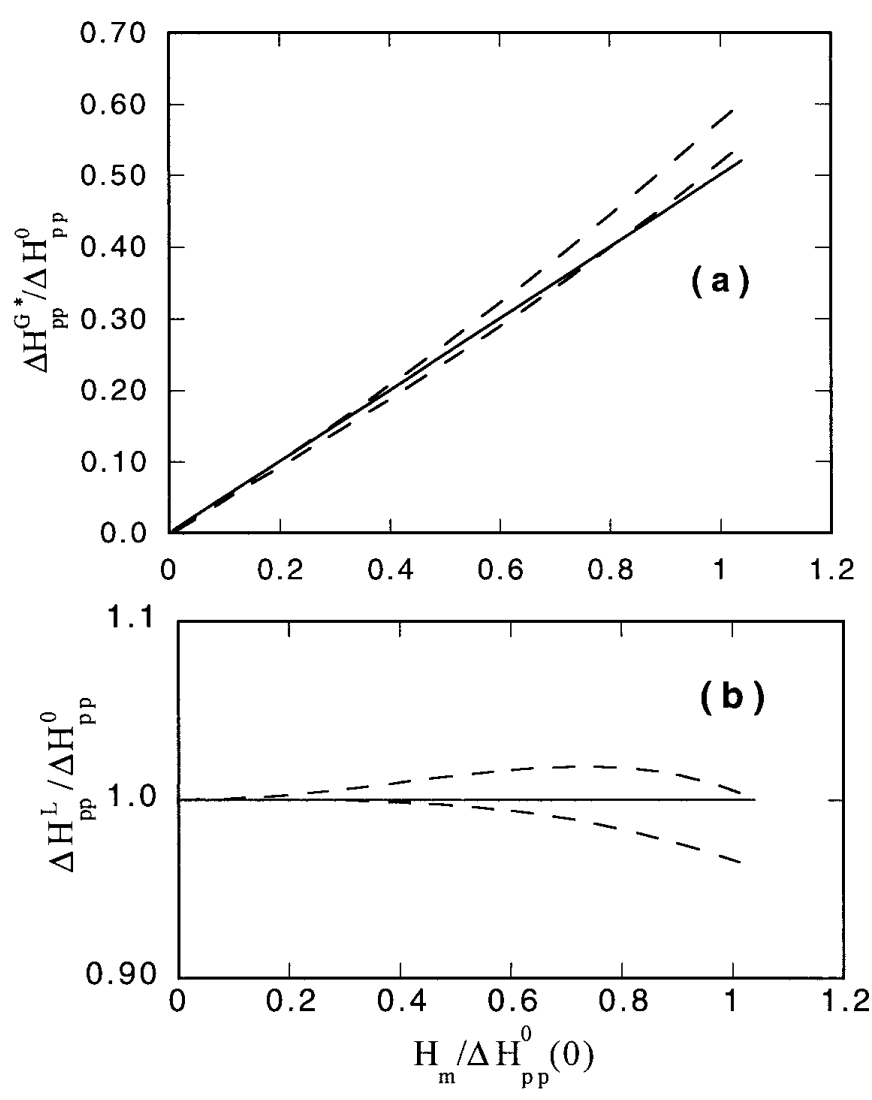

FIG. 1. (a) The additional Gaussian linewidth induced by field-modulation $\Delta H_{\mathrm{pp}}^{\mathrm{G} *}$ vs the peak-to-peak modulation amplitude normalized to the unbroadened linewidth. The upper dashed line corresponds to results measured from simulated spectra at $\chi=0.1$ and the lower to $\chi=4.0$, while the solid line is a plot of Eq. [8] with $\kappa=1 / 2$. Plots of Eq. [8] with $\kappa$ given by Eq. [9] are indistinguishable from the dashed lines. (b) The normalized Lorentzian linewidth vs the normalized peak-to-peak modulation amplitude. The upper dashed line corresponds to results measured from simulated spectra at $\chi=0.1$ and the lower to $\chi=4.0$, while the solid line is a plot of constant Lorentzian linewidth.

\section{RESULTS}

\section{Unresolved Hyperfine Structure}

Following Windle (16), we studied 3-carboxy proxyl in buffered aqueous solution and carbon tetrachloride in order to cover a wide range of solvent polarities.

Figure 2 shows the center line of the 3-carboxy proxyl EPR spectral line in $\mathrm{CCl}_{4}$ at $9.5^{\circ} \mathrm{C}$ using a deoxygenated sample and the following settings: power, $0.32 \mathrm{~mW} ; H_{\mathrm{m}}, 0.05 \mathrm{G}$; time constant, $10 \mathrm{~ms}$; and sweep time, $42 \mathrm{~s}$. The spectral line was fit to Eq. [3] using a fit window of $3.5 \Delta H_{\mathrm{pp}}^{0}$. The lower trace is the difference between the fit and the experimental spectral line showing that the sum function approximation to a Voigt, Eq. [3], is an excellent description of the observed spectral line. From this fit, and the universal map, Eqs. [17] and [10] of Ref. (8), we calculate that $\chi=4.61$. The overall linewidth of the spectral line in Fig. 2 is $\Delta H_{\mathrm{pp}}^{0}=1.195 \mathrm{G}$, and using the scheme in Fig. 11 of Ref. (8) leads to $\Delta H_{\mathrm{pp}}^{\mathrm{G}}=1.037 \mathrm{G}$ and $\Delta H_{\mathrm{pp}}^{\mathrm{L}}=$

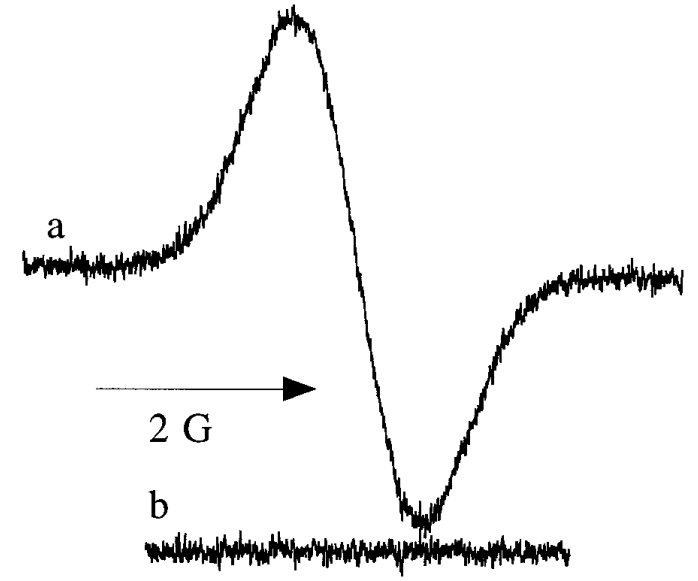

FIG. 2. (a) The center line of the EPR spectrum of 3-carboxy proxyl at $9.5^{\circ} \mathrm{C}$ in deoxygenated $\mathrm{CCl}_{4}$. (b) The difference between the experimental spectrum and the best fit to the sum function, Eq. [3].

$0.225 \mathrm{G}$. Measuring seven spectra leads to the mean values and standard deviations in Table 1. Note that the Gaussian component dominates the spectral line, having a linewidth that is $87 \%$ that of the entire line. Increasing the microwave power to 2.5 $\mathrm{mW}$ increases the Lorentzian linewidth by $0.03 \mathrm{G}$ and leaves the Gaussian linewidth unchanged. Averaging over seven spectra gives the results in Table 1.

The spectral line in Fig. 2 was taken at low power, modulation amplitude, and temperature (to reduce spin exchange) to optimize the resolution in an attempt to deduce the hyperfine coupling constant to the protons at the 4 position. There is no apparent incipient resolution in the spectral line, so we made an effort to detect this hyperfine coupling by fitting the spectral line to a model of a triplet of spectra of form Eq. [3] having relative intensities 1:2:1 separated by the proton hyperfine coupling constant at position $4, a_{H_{4}}$. The hyperfine spacing between members of the triplet was varied as a fit parameter. This yielded an excellent fit with $\Delta H_{\mathrm{pp}}^{\mathrm{G}}=0.895 \mathrm{G}$ and $a_{H_{4}}=$ $0.306 \pm 0.011$, where the error is the estimate derived from the

TABLE 1

H yperfine Coupling Constants, $G$ aussian and Lorentzian Linewidths for $3-\mathrm{C}$ arboxy Proxyl $\left(T=9.5^{\circ} \mathrm{C}\right)$

\begin{tabular}{llll}
\hline \multicolumn{1}{c}{ Solvent } & \multicolumn{1}{c}{$A_{0}, \mathrm{G}$} & \multicolumn{1}{c}{$\Delta H_{\mathrm{pp}}^{\mathrm{G}}(0), \mathrm{G}$} & \multicolumn{1}{c}{$\Delta H_{\mathrm{pp}}^{\mathrm{L}}, \mathrm{G}$} \\
\hline Hepes & $16.230 \pm 0.002$ & $1.045 \pm 0.003^{a}$ & $0.239 \pm 0.004^{b}$ \\
$\mathrm{CCl}_{4}$ & $14.128 \pm 0.001$ & $1.05 \pm 0.01^{a}$ & $1.49 \pm 0.02^{c}$ \\
$\mathrm{CCl}_{4}$ (deoxygenated) & & $1.038 \pm 0.006^{d}$ & $0.224 \pm 0.004^{e}$ \\
\hline
\end{tabular}

${ }^{a}$ Intercept of Eq. [5]. Mean values and standard deviations in three lines.

${ }^{b}$ Center line. Averaged over eight spectra at $H_{\mathrm{m}}=0.2$ to $1.2 \mathrm{G}$.

${ }^{c}$ Center line. Averaged over 18 spectra at $H_{\mathrm{m}}=0.25$ to $2.0 \mathrm{G}$.

${ }^{d}$ Mean values and standard deviations in 21 measurements, 3 lines each in 7 spectra $H_{\mathrm{m}}=0.05 \mathrm{G}$.

${ }^{e}$ Center line; mean values and standard deviations in seven spectra. 

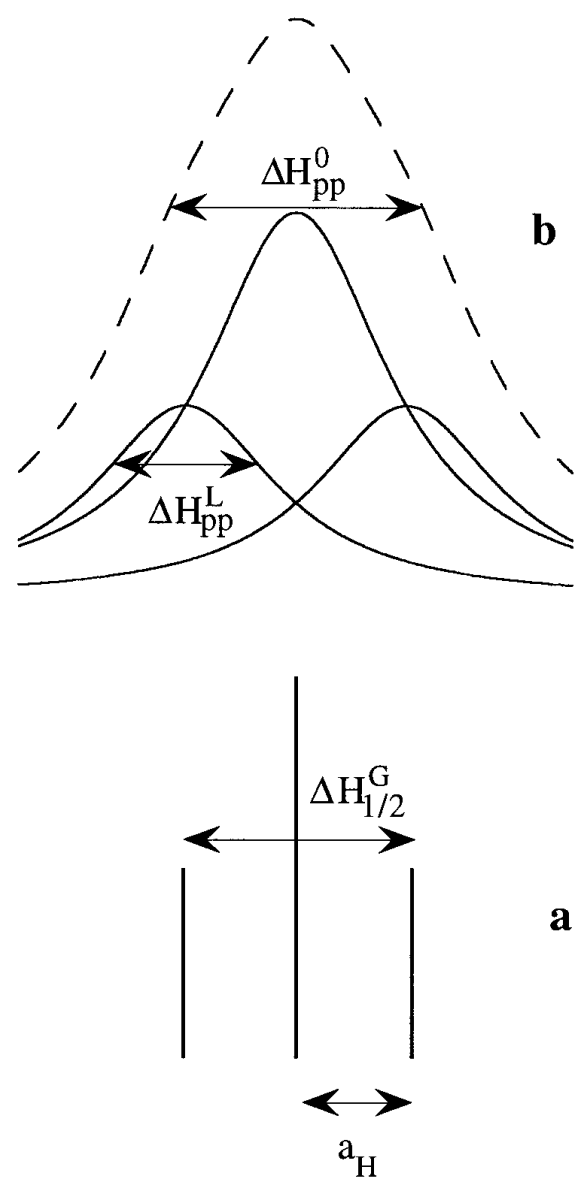

FIG. 3. (a) Stick pattern due to two equivalent protons, a triplet, spaced by the proton hyperfine coupling constant, $a_{H}$, of relative intensities 1:2:1. The width of the pattern at one-half maximum intensity is exactly the spacing of the outer hyperfine lines, $\Delta H_{1 / 2}^{\mathrm{G}}=2 a_{H}$. (b) Lorentzian lineshapes (solid lines) imposed upon the hyperfine pattern and the resultant sum of the three lines (dashed line).

nonlinear least-squares fitting in the standard fashion. Unfortunately, the difference between the residuals using a single sum function, Eq. [3], and those using the triplet is negligible; therefore, we learn that such a triplet of Voigts produces an excellent Voigt, so one is not enlightened whether the $a_{H_{4}}=$ $0.306 \mathrm{G}$ is the coupling to the two protons at position 4 or not. However, the two equally good fits gave us an unexpected opportunity to test the validity of a proposed method, p. 92 of Ref. (8), to find the factor $\alpha$ in Eq. [1].

The method, applied to two equivalent protons, $N=2$, is particularly straightforward since the pattern has lines of onehalf maximum intensity. The hyperfine pattern is a simple triplet of relative intensities 1:2:1 spaced by $a_{H}$ (Fig. 3a); thus, the full-width at half-maximum $\Delta H_{1 / 2}^{\mathrm{G}}=2 a_{H}$ as shown in Fig. 3a. Figure 3 is prepared in absorption presentation for clarity, so peak-to-peak linewidths appear between points of maximum slope. Figure $3 \mathrm{~b}$ shows lineshapes, in this case Lorentzians, imposed upon the stick pattern (solid lines) and the sum of the three lines to give the resultant spectrum (dashed lines). To convert to a peak-to-peak value of the Gaussian component, we use the fact that $\Delta H_{1 / 2}^{\mathrm{G}}=\sqrt{2 \ln 2} \Delta H_{\mathrm{pp}}^{\mathrm{G}}$; thus, $\Delta H_{\mathrm{pp}}^{\mathrm{G}}=2 a_{H} /$ $\sqrt{2 \ln 2}$. Setting this equal to the value computed from Eq. [1], $\Delta H_{\mathrm{pp}}^{\mathrm{G}}=\sqrt{2 \alpha} a_{H}$, yields $\alpha$ (theory) $=(2 \ln 2)^{-1}=1.44$.

In all of the cases discussed before Ref. (8), the number of protons was so large, yielding values of $\alpha$ so nearly equal to unity, that it was difficult to test the method. Further, previously (8) we had to interpolate between lines to find the position of half-maximum intensity. In this case, no interpolation is needed and $\alpha$ (theory) $=1.44$ is a substantial departure from unity and ought to provide a better test.

Before describing the test, we make some comments about Fig. 3b.

Lorentzian lineshapes were chosen to prepare Fig. $3 b$ because the figure nicely illustrates the linewidths that are pertinent to a Voigt. The Voigt (dashed line) has a Lorentzian component provided by the Lorentzian linewidth of the separate lines and a Gaussian component provided by the spread of the pattern. Thus, in Fig. 3b, the linewidth of the Lorentzian component of the Voigt (dashed line) is exactly equal to the linewidth of the separate lines, $\Delta H_{\mathrm{pp}}^{\mathrm{L}}$. The linewidth of the Gaussian component of the Voigt (dashed line) as calculated earlier is $2 a_{H} / \sqrt{2 \ln 2}$. With the choices of $a_{H}$ and $\Delta H_{\mathrm{pp}}^{\mathrm{L}}$ used in the preparation of Fig. $3 \mathrm{~b}$, the linewidth of the Voigt, $\Delta H_{\mathrm{pp}}^{0}$, is about 2.5 times larger than its Lorentzian component and about 1.3 times larger than its Gaussian component. In Fig. 3b, $\chi$ is about 2 .

Returning to the test of the method to evaluate $\alpha$, we observe that the case at hand is slightly more complicated than that illustrated in Fig. 3 in that the lineshapes imposed upon the three lines are themselves Voigts. In this case, the linewidth of the Lorentzian component of the resulting Voigt is the linewidth of the Lorentzian component of the separate Voigts. The linewidth of the Gaussian component of the resulting Voigt must be calculated by adding in quadrature the Gaussian linewidths from the separate lines to that due to the spread. Thus, the squared Gaussian linewidth of the experimental line treated as a triplet is given by $(0.895 \mathrm{G})^{2}+2 \alpha(0.306 \mathrm{G})^{2}$. This must be equal to the squared Gaussian linewidth of the experimental line treated as a singlet, $(1.037 \mathrm{G})^{2}$, from which we may calculate the experimental value of $\alpha$. This gives $\alpha$ (experiment $)=1.46$, very nearly equal to $\alpha$ (theory) $=1.44$. Carrying out these fittings and calculations for seven spectra yields $\alpha($ experiment $)=1.38 \pm 0.07$, the error being the standard deviation in the seven experiments.

For a spectral line such as that in Fig. 2, with a large value of $\chi$, the universal map becomes suspect; however, we are able to confirm its validity for 3-carboxy proxyl by studying an air-saturated sample. The spectral line (not shown) is broadened to an overall linewidth of $\Delta H_{\mathrm{pp}}^{0}=1.513 \mathrm{G}$ because of spin exchange interactions with dissolved oxygen (17). These spin exchange interactions do not affect the Gaussian component because the unresolved proton hyperfine pattern remains 


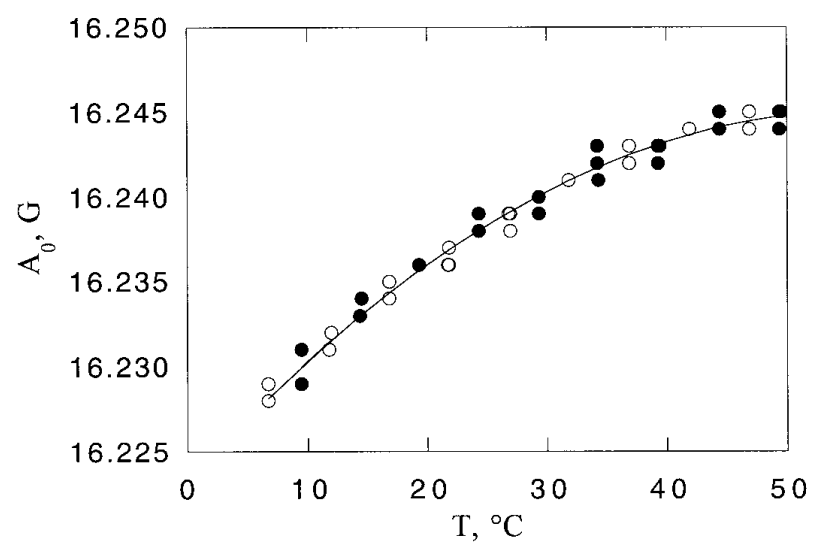

FIG. 4. The temperature dependence of the ${ }^{14} \mathrm{~N}$ hyperfine coupling constant for 3-carboxy proxyl in Hepes buffer, $\mathrm{pH}$ 7.4. The solid symbols mark data points taken with increasing temperature and the open symbols with decreasing temperature. The solid line is a plot of Eq. [10].

the same. Carrying out the same fitting procedure as that discussed in connection with Fig. 2, we find that the Lorentzian component is broadened to $\Delta H_{\mathrm{pp}}^{\mathrm{L}}=1.49 \pm 0.02 \mathrm{G}$ while the Gaussian component has a linewidth of $\Delta H_{\mathrm{pp}}^{\mathrm{G}}=1.05 \pm 0.01$. The reduced precision resulted because of an unexpected complication in the analysis of the air-saturated $\mathrm{CCl}_{4}$ data. The larger overall linewidth resulted in a larger fit window which led to inclusion of only a portion of the flanking ${ }^{13} \mathrm{C}$ lines. This led to errors in the determination of the mixing parameters and in turn in the values of $\chi$ and $\Delta H_{\mathrm{pp}}^{\mathrm{G}}$. Thus, a large fit window was employed that extended well beyond the ${ }^{13} \mathrm{C}$ lines. This resulted in a $1 \%$ change of the Gaussian linewidth that was taken to be the uncertainty. In the air-saturated sample, the increased Lorentzian linewidth lowers the Voigt parameter to $\chi$ $=0.705$, at which point all maps yield negligibly different values of $\Delta H_{\mathrm{pp}}^{\mathrm{G}}(8)$. The fact that $\Delta H_{\mathrm{pp}}^{\mathrm{G}}$ found for $\chi=4.61$ and 0.705 are equal within $1 \%$ (Table 1) demonstrates that the universal map is valid for 3-carboxy proxyl.

The hyperfine coupling constant to the ${ }^{14} \mathrm{~N}$ nucleus, $A_{0}$, was measured as one-half the separation of the high- and low-field lines as described in detail previously (18). The results are given in Table 1.

Measurements of $A_{0}, \Delta H_{\mathrm{pp}}^{\mathrm{G}}(0)$, and $\Delta H_{\mathrm{pp}}^{\mathrm{L}}$ in air-saturated Hepes buffer were similarly carried out and the results are tabulated in Table 1. Typical of nitroxide spin probes, $A_{0}$ varies substantially with polarity (19-22); however, the Gaussian linewidth is identical in the two solvents.

The variation of $A_{0}$ with temperature is shown in Fig. 4. The solid symbols mark data points taken with increasing temperature and the open symbols with decreasing temperature. The solid line is a least-squares fit of a quadratic function of the temperature to all of the data yielding

$$
A_{0}(T)=16.223+7.75 \times 10^{-4} T-6.88 \times 10^{-6} T^{2},
$$

where $A_{0}(T)$ is given in gauss when $T$ is given in ${ }^{\circ} \mathrm{C}$ valid in the range $T=5-50^{\circ} \mathrm{C}$. This level of precision is needed in problems involving changes in $\mathrm{pH}$ where $A_{0}$ decreases by about 0.2 $\mathrm{G}$ when the molecule is protonated at low values of $\mathrm{pH}$ (23). Also, high precision is needed if the spin exchange frequency is to be deduced from the shift of the ${ }^{14} \mathrm{~N}$ hyperfine lines (24).

The Gaussian linewidth varies linearly with temperature in the range $7-50^{\circ} \mathrm{C}$ (not shown) as

$$
\Delta H_{\mathrm{pp}}^{\mathrm{G}}(T)=1.057-1.20 \times 10^{-3} T, \quad r=0.998,
$$

where $\Delta H_{\mathrm{pp}}^{\mathrm{G}}(T)$ is given in gauss when $T$ is given in ${ }^{\circ} \mathrm{C}$ and $r$ is the coefficient of correlation.

\section{Modulation Broadening}

Figure 5 shows the Gaussian linewidth as a function of the peak-to-peak modulation amplitude for 3-carboxy proxyl in aqueous Hepes buffer (solid symbols) and in air-saturated $\mathrm{CCl}_{4}$, (open symbols). The data in deoxygenated $\mathrm{CCl}_{4}$ are the same within experimental error to those in the aqueous buffer. The data in Fig. 5 extend up to values of $H_{\mathrm{m}}$ equal to the unbroadened overall linewidth of the spectra; thus, higher values of $H_{\mathrm{m}}$ are employed with the air-saturated $\mathrm{CCl}_{4}$ sample. The two data sets are at quite different values of the Voigt parameter: near $\chi=4.6$ for the Hepes and near $\chi=0.7$ for the air-saturated $\mathrm{CCl}_{4}$. The solid line is a plot of Eq. [5] with $\Delta H_{\mathrm{pp}}^{\mathrm{G}}(0)=1.04 \mathrm{G}$ and $\kappa=1 / 2$ and is seen to describe the modulation broadening of the low-valued $\chi$ extremely well and even the high-valued $\chi$ rather well. At the maximum value of modulation amplitude, $H_{\mathrm{m}} \approx \Delta H_{\mathrm{pp}}^{0}(0)$, the measured values of $\Delta H_{\mathrm{pp}}^{\mathrm{G}}\left(H_{\mathrm{m}}\right)$ for the Hepes sample are about 3\% smaller than those calculated from Eq. [5], a negligible difference in view of

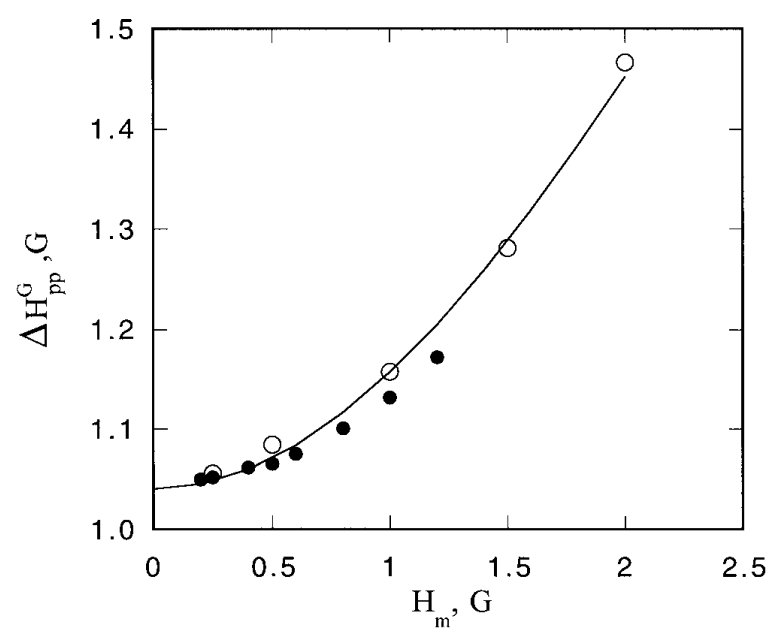

FIG. 5. The Gaussian linewidth versus the peak-to-peak modulation amplitude for 3-carboxy proxyl in aqueous Hepes buffer, solid symbols, and in air-saturated $\mathrm{CCl}_{4}$, open symbols. The solid line as a plot of Eq. [5] with $\Delta H_{\mathrm{pp}}^{\mathrm{G}}(0)=1.04 \mathrm{G}$ and $\kappa=1 / 2$. 
the various uncertainties involved in the corrections (8). Within experimental error, the Lorentzian linewidth was constant as a function of $H_{\mathrm{m}}$ for each of the samples. Averaging over all values of $H_{\mathrm{m}}$ yielded the values given in Table 1 . The complication in the analysis of the air-saturated $\mathrm{CCl}_{4}$ data due to the ${ }^{13} \mathrm{C}$ lines discussed earlier led to uncertainties in Gaussian linewidth that varied from $1.5 \%$ at $H_{\mathrm{m}}=0.2 \mathrm{G}$ up to $4.5 \%$ at $H_{\mathrm{m}}=1.8 \mathrm{G}$.

\section{DISCUSSION}

A clear, quantitative understanding of the effect of field modulation on a Voigt line is now available. Only the Gaussian component is broadened and this broadening may be calculated to first order from Eq. [5]. Since such Gaussian broadening may be corrected, yielding accurate values of $\Delta H_{\mathrm{pp}}^{\mathrm{L}}$, modulation broadening may be used to increase the signal-to-noise for weak signals with no significant loss in information. The effect upon the time required for an experiment can be rather significant. For example, if one increases $H_{\mathrm{m}}$ from about $0.1 \Delta H_{\mathrm{pp}}^{0}$ to $1.0 \Delta H_{\mathrm{pp}}^{0}$, realizing about a factor of 10 in improved signal-tonoise, the number of scans may be reduced by 100 . Reducing accumulation time by two orders of magnitude can make the difference in the feasibility of an experiment. Almost all spin probes produce spectra well described by the Voigt shape; thus, the effect of modulation may be predicted a priori in order to plan spin-probe investigations.

The validity of Eq. [5] is demonstrated experimentally for 3-carboxy proxyl by the results in Fig. 5. The spin probe 3-carboxy proxyl yields EPR spectra with severe inhomogeneous broadening leading to large values of the Voigt parameter in some cases. The Gaussian linewidth arising from unresolved hyperfine structure is the same in aqueous buffer and $\mathrm{CCl}_{4}$, so previous methods (8) to correct for inhomogeneous broadening may be applied without reference to the solvent. Similarly to the doxyl-labeled alkyl chains $(8,9)$, large values of $\chi$ are encountered even though the spectral line remains unresolved; therefore, detailed knowledge of the proton hyperfine coupling constants cannot be obtained from EPR measurements alone. ${ }^{2}$ Failure to correct the observed linewidth to find $\Delta H_{\mathrm{pp}}^{\mathrm{L}}$ would result in errors of about $400 \%$ for either Hepes buffer or the deoxygenated $\mathrm{CCl}_{4}$ solutions and $25 \%$ for airsaturated $\mathrm{CCl}_{4}$ solutions. Now that the proxyl moiety has been studied, the Gaussian linewidths of all of the major spin probes are now available $(8,9)$ and the methods of Ref. $(8)$ may be used to correct spin label data to first order; however, Gaussian linewidths vary for a variety of reasons, including spin exchange (8). Therefore, precision work will continue to require a determination of the Gaussian linewidths in each experiment.

In addition to the motivation to increase the signal-to-noise ratio, there may be cases in which it would be beneficial to

\footnotetext{
${ }^{2}$ These coupling constants could be measured using NMR if need be. See Ref. (9) and references therein.
}

TABLE 2

Calculated Values of $\alpha$ in Eq. [1]

\begin{tabular}{cc}
\hline $\begin{array}{c}\text { Number of equivalent } \\
\text { protons }\end{array}$ & $\alpha$ \\
\hline 2 & 1.443 \\
4 & 1.282 \\
6 & 1.164 \\
8 & 1.105 \\
10 & 1.078 \\
12 & 1.081 \\
14 & 1.076 \\
16 & 1.067 \\
18 & 1.056 \\
\hline
\end{tabular}

Note. According to p. 92 of Ref. (8).

modulation-broaden the lines intentionally. One such case occurs in problems employing spin exchange of spin probes in liquids. At spin exchange frequencies comparable with the Gaussian linewidth (in frequency units), the spin exchange tends to collapse the hyperfine structure $(8,25,26)$. Thus, there is a troublesome region of spin exchange frequencies in which the linewidth of the Gaussian component decreases with spin exchange, complicating the correction procedures. This has been solved $(8,25)$; however, it is complicated. A straightforward experimental solution to the problem is to increase the modulation amplitude until the modulation-induced Gaussian component is large compared with the unresolved hyperfine coupling component. In this way, a constant Gaussian component may be easily corrected.

Typical of nitroxide spin probes, the ${ }^{14} \mathrm{~N}$ hyperfine coupling constant, $A_{0}$, for 3-carboxy proxyl varies substantially with polarity (19-22) (Table 1); however, the Gaussian linewidth is identical in the two solvents (Table 1), obviating the need to adjust this quantity for solvent polarity. This does not mean that the proton hyperfine coupling constants are independent of polarity, only that the sum in Eq. [1] does not vary. For those spin probes that have been studied in detail $(8,9)$, the changes in Gaussian linewidth with polarity vs that of $A_{0}$ are uncorrelated. For example, in changing solvents from water to $\mathrm{CCl}_{4}$, the Gaussian linewidth changes in the $80-90 \%$ range for TEMPONE and DTBN, in the $10 \%$ range for TEMPO, TEMPAMINE, and CTPO, and less than 5\% for the DOXYL labeled alkyl chains and TEMPOL. ${ }^{3}$ Now we find that 3 -carboxy proxyl joins this latter group.

The fact that the experimental value of $\alpha=1.38 \pm 0.07$ is within experimental error of the theoretical value of $\alpha$ (theory) $=1.44$ demonstrates that the previously proposed method to calculate this quantity on p. 92 of Ref. (8) is valid. Table 2

\footnotetext{
${ }^{3}$ Acronyms: TEMPO, 2,2,6,6-tetramethylpiperidine- $N$-oxyl; TEMPONE, 4-oxo-TEMPO; TEMPAMINE, 4-amino-TEMPO; TEMPOL, 4-hydroxyTEMPO; DTBN, di-tert-butylnitroxide; CTPO, 3-carbamoyl-2,2,5,5-tetramethyl-3-pyrroline-1-yloxyl; DOXYL, 4,4-dimethyloxazolidine- $N$-oxyl.
} 
gives the results of the calculations of $\alpha$ as a function of the number of interacting protons.

\section{APPE NDIX}

\section{Transformation of $\eta_{A}$ to $\chi$ at Small Values of $\chi$}

At small values of $\chi$, the sum function, Eq. [3], is an excellent approximation to the Voigt line shape. For example, at $\chi=0.001$, the sum function reproduces the Voigt to within a maximum fractional deviation of $2 \times 10^{-4}$. Simulating Voigt lines and carrying out fittings as described in Ref. (12) over a fit window $4.0 \Delta H_{\mathrm{pp}}^{0}$, we arrive at the following:

$$
\chi=\sqrt{2.7721\left(1-\eta_{A}\right)+13.937\left(1-\eta_{A}\right)^{2}},
$$

valid over the region $0<\chi<0.5$. Over this region, the map in Eq. [12] reproduces the true value of $\chi$ to within 0.005 while the Voigt $(8,12)$ and universal maps $(8)$ yield values of $\chi$ in error by as much as 0.1 .

\section{ACKNOWLEDGMENTS}

This work was supported by grants from the Fundacão de Amparo À Pesquisa do Estado de São Paulo (FAPESP), the Conselho Nacional de Desenvolvimento Científico e Tecnológico, the NIH/MBRS S06 GM4868003, the NIH 1 R15 GM/HL51103-01A1, and the CSUN Research and Grants Committee. FAPESP also provided a travel grant to BB.

\section{REFERENCES}

1. C. P. J . Poole, "Electron Spin Resonance: A Comprehensive Treatise on Experimental Technique." Wiley, New York (1983).

2. J. S. Hyde, P. B. Sczaniecki, and W. Froncisz, J. Chem. Soc., Faraday Trans. 1 85, 3901 (1989).
3. P. B. Sczaniecki, J. S. Hyde, and W. Froncisz, J . Chem. Phys. 93, 3891 (1990).

4. P. B. Sczaniecki, J . S. Hyde, and W. Froncisz, J . Chem. Phys. 94, 5907 (1991).

5. H. S. Mchaourab and J. S. Hyde, J. Chem. Phys. 98, 1786 (1993).

6. B. L. Bales and M. E. Baur, Chem. Phys. Letters 7, 341 (1970).

7. M. Peric and H. J. Halpern, J. Magn. Reson. A 109, 198 (1994).

8. B. L. Bales, in "Biological Magnetic Resonance" (L. J . Berliner and J. Reuben, Ed.), Vol. 8, p. 77. Plenum, New York (1989).

9. B. L. Bales, D. Mareno, and F. L. Harris, J . Magn. Reson. A 104, 37 (1993).

10. B. L. Bales, D. Mareno, R. Blum, H. J. Halpern, and M. Peric, J. Magn. Reson. 98, 299 (1992).

11. B. L. Bales, J. Mag. Reson. 48, 418 (1982).

12. H. J. Halpern, M. Peric, C. Yu, and B. L. Bales, J. Magn. Reson. 103, 13 (1993).

13. G. K. Wertheim, M. A. Butler, K. W. West, and D. N. E. Buchanan, Rev. Sci. 45, 1369 (1974).

14. H. Wahlquist, J. Chem. Phys. 35, 1708 (1961).

15. S. N. Dobryakov and Y. S. Lebedev, Sov. Phys.-Doklady 13, 873 (1969).

16. J . J . Windle, J. Magn. Reson. 45, 432 (1981).

17. J. S. Hyde and W. K. Subczynski, J. Magn. Reson. 56, 125 (1984).

18. B. L. Bales, E. Wajnberg, and O. R. Nascimento, J . Magn. Reson. A 118, 227 (1996).

19. B. R. Knauer and J . J. Napier, J . Am. Chem. Soc. 98, 4395 (1976).

20. T. Abe, S. Tero-Kubota, and Y. Ikegami, J. Phys. Chem. 86, 1358 (1982).

21. A. H. Reddock and S. Konishi, J. Chem. Phys. 70, 2121 (1979).

22. N. A. Malik, E. A. Smith, and M. C. R. Symons, J. Chem. Soc., Faraday Trans. 1 85, 3245 (1989).

23. R. N. Schwartz, M. Peric, S. A. Smith, and B. L. Bales, J. Phys. Chem. B 101, 8735 (1997).

24. B. L. Bales and M. Peric, J. Phys. Chem. B 101, 8707 (1997).

25. B. L. Bales, J. Magn. Reson. 38, 193 (1980).

26. W. Plachy and D. Kivelson, J. Chem. Phys. 47, 3312 (1967). 